\section{Monitoramento por entrevistas telefônicas de fatores de risco para doenças crônicas: experiência de Goiânia, Goiás, Brasil}

\author{
Surveillance of risk factors for chronic diseases \\ through telephone interviews: experience in \\ Goiânia, Goiás State, Brazil
}

\footnotetext{
${ }^{1}$ Faculdade de Nutrição, Universidade Federal de Goiás, Goiânia, Brasil. 2 Secretaria Estadual de Saúde de Goiás, Goiânia, Brasil.

3 Programa de PósGraduação em Nutrição Humana, Universidade de Brasília, Brasília, Brasil. ${ }^{4}$ Núcleo de Pesquisas Epidemiológicas em Nutrição e Saúde, Universidade de São Paulo, São Paulo, Brasil.

5 Centro de Ciências da Saúde, Universidade Federal do Pará, Belém, Brasil.

Correspondência M. R. G. Peixoto

Faculdade de Nutrição, Universidade Federal de Goiás.

Rua 49, Quadra C-2, Lote 2 Goiânia, GO

74810-039, Brasil.

hbpeixoto@uol.com.br
}

\begin{abstract}
This study describes results from a surveillance system for risk factors for chronic non-communicable diseases in 2005 in the city of Goiânia, Goiás State, Brazil. A probabilistic sample ( $n=$ 2,002) of the adult population living in households with landline telephones was studied by phone interviews. Factors investigated were: food consumption, physical activity, smoking, alcohol intake, self-reported weight and height, and self-reported medical diagnosis of chronic non-communicable diseases. Prevalence and $\chi^{2}$ values were calculated. The data showed: low consumption (< 5 days/week) of fruits and vegetables (47.1\%), high rate of physical inactivity at work (86.6\%), in commuting (92.6\%), and during leisure time (61.9\%), high alcohol consumption (36.5\%), and high rates of obesity (10.6\%), hypertension (22.4\%), dyslipidemia (18.4\%), and diabetes (4.4\%). Most of the factors were inversely related to schooling and directly related to age $(p<0.05)$. High prevalence of risk factors for chronic non-communicable diseases was reported. The advantages of this system were: low operational cost and the ability to monitor trends in chronic non-communicable diseases at the local level.
\end{abstract}

Monitoring; Chronic Disease; Risk Factors; Interviews; Telephone
Maria do Rosário Gondim Peixoto ${ }^{1}$ Estelamaris Tronco Monego 1 Veruska Prado Alexandre 2,3 Rávila Graziany Machado de Souza 1 Erly Catarina de Moura 4,5

Introdução

Estudos epidemiológicos comprovam a associação entre doenças crônicas não transmissíveis e fatores de risco tais como a obesidade, inatividade física, consumo excessivo de álcool, tabagismo e padrões de consumo alimentar inadequados. Dentre estes últimos, a maior evidência situa-se no baixo consumo de frutas e hortaliças e no excesso de gorduras saturadas 1,2 .

Estimativas da Organização Mundial da Saúde (OMS) mostram que as doenças crônicas não transmissíveis são responsáveis por $58,5 \%$ da mortalidade e $45,9 \%$ do total de morbidades, reduzindo os anos de vida saudável 3 .

Apenas mais recentemente, estudos têm demonstrado que as características qualitativas da dieta influenciam no aparecimento das doenças crônicas não transmissíveis, em todos os momentos do curso da vida 4,5,6,7.

A tendência da evolução dos padrões de consumo alimentar brasileiro nas últimas três décadas, representativo das áreas metropolitanas do país, indica que nas regiões economicamente mais desenvolvidas e, de forma geral, no meio urbano e entre as famílias com maior rendimento há um consumo excessivo de açúcar e gorduras, em especial do tipo saturada, associado ao consumo insuficiente de frutas e hortaliças (Instituto Brasileiro de Geografia e Estatística. http://www.ibge.gov.br, acessado em 10/Abr/2005) 
Apesar do grande intervalo de tempo que separa a realização de inquéritos domiciliares sobre condições de saúde e nutrição dos brasileiros, tais estudos têm revelado crescimento acelerado do sobrepeso e da obesidade, em particular entre a população de menor renda ${ }^{8}$.

A obesidade é considerada um problema de saúde pública de importância relevante, tanto em países desenvolvidos como em desenvolvimento, ocorrendo concomitantemente à diminuição progressiva do gasto energético em atividades laborais, no lazer, no cumprimento de afazeres domésticos e como decorrência de modificações no padrão alimentar 9,10.

O tabagismo e o consumo de bebidas alcoólicas estão entre os principais responsáveis pela carga de doenças no mundo. $\mathrm{O}$ tabagismo, em todas as suas formas, aumenta o risco de morbidade e mortes prematuras por doença coronariana, hipertensão arterial, acidente vascular encefálico, câncer e doenças pulmonares. Já o consumo de bebidas alcoólicas, quando excessivo, pode provocar problemas de saúde como hipertensão arterial, infarto e certos tipos de cânceres, além de inúmeras conseqüências sociais 1,11 .

Embora muito relevantes na definição do perfil epidemiológico de uma população, cujo monitoramento permite uma intervenção precoce, esses fatores de risco para doenças crônicas não transmissíveis não vêm sendo monitorados de forma adequada no Brasil 8 .

É possível conhecer o perfil de saúde de uma população mediante metodologia que preveja a auto-informação. Este procedimento permite avaliar amostras representativas da população, muitas vezes por um longo período de tempo com menor custo e simplificação do trabalho de campo ${ }^{12}$. O sistema de monitoramento por meio de entrevistas telefônicas reúne características potenciais de simplicidade, baixo custo e rapidez. No Brasil, este sistema vem sendo utilizado com sucesso em populações nas quais os serviços de telefonia alcançam a grande maioria das residências, situação que pode ser projetada para a maioria das cidades brasileiras num prazo relativamente curto 13 .

O presente artigo descreve métodos e resultados obtidos junto à população residente do Município de Goiânia, capital do Estado de Goiás, referente à experiência de implantação do Sistema Municipal de Monitoramento de Fatores de Risco para Doenças Não Transmissíveis a partir de Entrevistas Telefônicas (SIMTEL).

\section{Material e métodos}

O SIMTEL foi implantado no Brasil no ano de 2003, no Município de São Paulo, e no ano de 2005 foi novamente realizado nesta capital e expandido para outras capitais brasileiras (Belém - Pará, Florianópolis - Santa Catarina, Goiânia - Goiás e Salvador - Bahia). O sistema baseia-se na aplicação de inquéritos periódicos realizados em amostra probabilística da população adulta residente em domicílios servidos por linhas telefônicas fixas. No presente estudo foram analisados os dados referentes à cidade de Goiânia.

A seleção dos indivíduos para a participação no sistema de monitoramento (entrevista) foi realizada em duas etapas. A primeira compreendeu o sorteio sistemático e estratificado por regiões (centro, norte, sul, leste e oeste) de 7 mil linhas telefônicas residenciais fixas, de um universo de 307.301 linhas do cadastro eletrônico da empresa Brasil Telecom S.A. A seguir, as $7 \mathrm{mil}$ linhas sorteadas foram re-sorteadas e divididas em vinte réplicas com 350 linhas ordenadas de 1 a 20, cada réplica reproduzia a mesma proporção de linhas por região da cidade. A segunda etapa correspondeu ao sorteio aleatório simples de um morador com pelo menos 18 anos de idade para cada linha sorteada após relação nominal de todos os moradores adultos residentes no domicílio contatado.

No Município de Goiânia, a seleção das linhas telefônicas, seguido pelo sorteio dos moradores e a realização das entrevistas foram realizadas no período entre maio e dezembro de 2005, buscando alcançar o número mínimo de 2 mil entrevistas. Estimando, dessa forma, a prevalência de fatores de risco nesta população com um nível de significância de $5 \%$ e margem de erro de estimativa entre $2 \%$ e $3 \%$.

As entrevistas telefônicas foram realizadas com o auxilio de um microcomputador, mediante uso de um programa especialmente desenvolvido para o SIMTEL que permitia o sorteio do entrevistado e o registro das respostas diretamente na tela utilizando o teclado ou mouse. As entrevistas tinham uma duração média de oito minutos e incluíam a leitura na tela do computador de um conjunto de perguntas curtas e simples com alternativas pré-determinadas, registro das respostas, salto de réplicas de perguntas não aplicáveis de acordo com a resposta do entrevistado, gravação das entrevistas para posterior controle de qualidade e a criação automática de um banco de dados.

Cada número de telefone sorteado recebeu até dez ligações em dias e horários variados para exclusão de linhas não elegíveis (linhas fora de serviço, permanentemente sem resposta ou 
empresariais) ou para obtenção de aquiescência familiar para o estudo e sorteio do individuo a ser entrevistado. As entrevistas foram realizadas logo após o sorteio ou, no caso de ausência do sorteado, em dia e horário de sua conveniência. Maiores detalhes sobre os procedimentos metodológicos empregados no processo de amostragem e na operação do SIMTEL estão descritos em publicação anterior 13 .

As perguntas incluídas no questionário versavam sobre características demográficas e sócio-econômicas dos entrevistados e de suas famílias, padrões de alimentação e de atividade física, hábito de fumar e consumo de bebidas alcoólicas, peso e altura referidos, auto-avaliação do estado de saúde e referência a diagnóstico médico de doenças não transmissíveis, tais como diabetes, hipertensão arterial, dislipidemias e osteoporose.

Os indicadores selecionados do SIMTEL/ Goiânia envolveram variáveis comportamentais (consumo alimentar, atividade física, tabagismo e consumo de bebida alcoólica), peso, altura e referência a diagnóstico médico anterior de hipertensão arterial, diabetes e/ou dislipidemia (hipercolesterolemia).

Dados referentes ao consumo alimentar foram obtidos por meio da freqüência alimentar simplificada, sendo abordadas questões referentes ao consumo de frutas, hortaliças cruas e cozidas, feijão, refrigerantes, leite e carnes (ave e bovina). Neste estudo serão apresentados os dados do consumo de frutas e hortaliças.

A categoria de resposta sim/não identificava o consumo de frutas e hortaliças maior que cinco vezes/semana, considerado neste estudo como consumo regular. Se a resposta fosse afirmativa buscava-se identificar o número de vezes de ingestão desses alimentos ao dia.

Com relação ao consumo de bebida alcoólica, foi considerado consumo grave de álcool, independentemente do sexo, a ingestão de cinco doses de qualquer bebida alcoólica em pelo menos um dia do último mês e consumo de risco a ingestão superior a uma dose para mulher e duas doses para homem associado a uma freqüência $\geq 1$ dia da semana 14 .

O sedentarismo foi caracterizado pela ausência do exercício físico no lazer, auto-relato de esforço leve ou muito leve no trabalho, deslocamento motorizado para o trabalho e ausência de realização de atividades domésticas. Para a identificação do hábito de fumar atual ou anterior, utilizaram-se as categorias de resposta: sim/não.

As medidas antropométricas - peso e altura referidos - foram utilizadas para a determinação do índice de massa corporal (IMC), calculado pela razão do peso $(\mathrm{kg})$ pelo quadrado da altura $\left(\mathrm{m}^{2}\right)$. Para a análise, foi utilizada a recomendação da OMS 15 .

Informações sobre referência a diagnóstico médico anterior de hipertensão arterial, diabetes mellitus e dislipidemia foram obtidas por intermédio das categorias de resposta: sim/não.

O processamento de dados e as análises estatísticas foram realizados com o auxílio do aplicativo Statistical Software for Professionals, versão 7.0 (Stata Corp., College Station, Estados Unidos), empregando-se as funções "svy" que consideram a natureza complexa da amostra.

Inicialmente foi calculada a distribuição da população adulta de Goiânia com telefone, confrontando-se com os dados da população total do município (IBGE. Censo Demográfico 2000. http://www.ibge.gov.br). Conforme descrito por Monteiro et al. 13, foram calculadas estimativas da prevalência dos indicadores para a população adulta do município com telefone e para a população adulta total do município. No primeiro caso, as estimativas foram produzidas empregando-se fatores individuais de ponderação correspondentes ao número de adultos no domicílio do entrevistado multiplicado pelo inverso do número de linhas telefônicas.

No segundo caso, multiplicou-se o fator individual de ponderação por um fator adicional que levou em conta diferenças sócio-demográficas existentes entre a população adulta com telefone e a população adulta total do município. Para obtenção desse fator adicional de ponderação, a amostra da população com telefone (estudada pelo sistema de monitoramento e já incorporando o fator de ponderação relativo ao número de adultos e de linhas telefônicas em cada domicílio) foi comparada a uma amostra da população do município, proveniente do mais recente Censo Demográfico (10\% dos domicílios estudados em 2000). Tal comparação foi feita com base na estratificação das duas amostras segundo sexo (masculino e feminino), seis faixas etárias (18-24, 25-34, 35-44, 45-54, 5564 e 65 e mais anos de idade) e quatro níveis de escolaridade (0-4, 5-8, 9-11 e 12 ou mais anos de escolaridade). A seguir, calculou-se, para cada um dos 48 estratos constituídos, a razão observada entre freqüência do estrato na amostra do Censo Demográfico e freqüência do estrato na amostra do sistema de monitoramento. Esta razão veio a ser o fator adicional de ponderação que, aplicado aos indivíduos de cada estrato, corrigiu a eventual sub ou super-representação de homens ou mulheres, faixas etárias ou estratos sociais na amostra de adultos estudada pelo sistema de monitoramento vis-à-vis a representação dos mesmos na população adulta total do município 13 . 
Foram calculadas médias com desvio-padrão e porcentagens com intervalos de confiança (IC95\%), para homens e mulheres, segundo o ajuste para representar a população adulta do município com telefone. As análises estatísticas da associação entre os fatores de risco e as variáveis sócio-demográficas como sexo foram realizadas pelo emprego do teste estatístico de distribuição do qui-quadrado. Foi utilizado o nível de significância de $5 \%$.

Este projeto foi analisado e aprovado pelo Comitê de Ética em Pesquisa da Faculdade de Saúde Pública da Universidade de São Paulo de acordo com requisitos da Resolução no. 196/96 do Conselho Nacional de Saúde. Por se tratar de entrevista por telefone, o Termo de Consentimento Livre e Esclarecido foi substituído pelo consentimento verbal obtido por ocasião dos contatos telefônicos e devidamente gravado.

\section{Resultados}

Para a obtenção do número mínimo de entrevistas (2 mil), foram utilizadas nove réplicas, totalizando 3.145 linhas telefônicas, sendo elegíveis $2.738(87,1 \%)$. As principais causas de não-elegibilidade foram: linhas permanentemente sem respostas ou que não respondiam após dez cha-

Tabela 1

Distribuição percentual da população adulta e da população adulta com telefone segundo variáveis sócio-demográficas. Goiânia, Goiás, Brasil, 2000/2005.

\begin{tabular}{lcc}
\hline Variável & $\begin{array}{c}\text { População adulta } \\
\text { (2000) }\end{array}$ & $\begin{array}{c}\text { População adulta } \\
\text { com telefone (2005) }\end{array}$ \\
\hline Sexo & & \\
Masculino & 46,6 & 37,6 \\
Feminino & 53,4 & 62,4 \\
Faixa etária (anos) & & 16,6 \\
18-24 & 23,2 & 21,9 \\
$25-34$ & 26,3 & 22,5 \\
$35-44$ & 21,5 & 18,8 \\
$45-54$ & 14,2 & 10,7 \\
$55-64$ & 8,1 & 9,5 \\
65 e + & 6,6 & 17,7 \\
Escolaridade (anos) & & 18,9 \\
0-4 & 26,3 & 38,8 \\
$5-8$ & 25,8 & 24,5 \\
$9-11$ & 31,8 & \\
12 e + & 16,1 & \\
\hline
\end{tabular}

Fonte: amostra de $10 \%$ dos domicílios do Censo Demográfico para a população adulta em 2000 e amostra estudada pelo SIMTEL $(n=2.002$ ) para população adulta com telefone em 2005. madas $(10,1 \%)$ e linhas fora de serviço ou inexistentes $(2,8 \%)$. Foram realizadas entrevistas em 2002 linhas telefônicas, definindo uma taxa de sucesso de $73,1 \%$. As principais causas que impediram a realização das entrevistas foram a recusa $(6,8 \%)$ ou linhas que não se conseguiu contato após dez chamadas $(4,4 \%)$ realizadas em dias e períodos variados. A duração média de cada entrevista foi de $8,2 \pm 2,7$ minutos.

Comparando-se as características sócio-demográficas dos adultos com telefones estudados pelo SIMTEL/Goiânia com as características da população adulta de Goiânia, apresentada pelo Censo Demográfico 2000, observa-se que a amostra estudada pelo sistema de monitoramento possui maior proporção de mulheres (62,4\% contra $53,4 \%$ no censo), menor proporção de jovens entre 18 e 24 anos de idade (16,6\% contra $23,2 \%)$ e maior proporção de indivíduos com escolaridade igual ou superior a nove anos $(38,8 \%$ contra $31,8 \%$ no censo - Tabela 1). As médias de idade e de anos de escolaridade para a população estudada pelo sistema de monitoramento foram de $41,3( \pm 16,0)$ e 9,6 anos $( \pm 4,5)$, respectivamente.

A Tabela 2 apresenta estimativas para a freqüência de fatores de risco selecionados de acordo com dois procedimentos de ajuste, um relativo à população adulta servida por linhas telefônicas e outro extensível à população adulta total no Município de Goiânia. Observa-se que as diferenças entre as duas alternativas de estimativa são relativamente pequenas e não sistemáticas: na maioria dos casos as diferenças absolutas não ultrapassaram cinco pontos percentuais para mais ou para menos, e as diferenças relativas ficaram abaixo de $15 \%$.

A Tabela 3 mostra a freqüência de fatores de risco e proteção para doenças crônicas não transmissíveis relativas à população masculina $\mathrm{e}$ feminina adulta com telefone. Foram observadas diferenças significativas entre os sexos quanto à freqüência da maioria dos fatores de risco estudados. Destaca-se uma menor freqüência de atividade física no lazer $(32,9 \%)$ e no trabalho $(11,2 \%)$ para as mulheres, no entanto, elas apresentaram um maior consumo regular de frutas e hortaliças $(52,2 \%)$. Já o consumo de bebida alcoólica em excesso $(37,7 \%)$ e o tabagismo $(15,1 \%)$ foram mais freqüentes entre os homens.

Com relação à auto-referência de doenças, as prevalências de obesidade e diabetes foram semelhantes entre homens e mulheres. O excesso de peso foi mais freqüente para os homens, enquanto a dislipidemia e a hipertensão arterial foram mais reportadas pelas mulheres (Tabela 3 ).

Para os homens, as freqüências do sedentarismo, excesso de peso e hipertensão aumentaram com a idade, já o consumo inadequado 
Estimativas para freqüência de fatores de risco e proteção para doenças crônicas não transmissíveis na população adulta segundo procedimentos de ajuste. Goiânia, Goiás, Brasil, 2005.

\begin{tabular}{|c|c|c|c|c|}
\hline \multirow[t]{2}{*}{ Fatores } & \multicolumn{2}{|c|}{$\begin{array}{l}\text { Freqüência segundo ajuste } 1 \text { * } \\
\qquad(n=2.002)\end{array}$} & \multicolumn{2}{|c|}{$\begin{array}{l}\text { Freqüência segundo ajuste } 2 \text { ** } \\
\qquad(n=2.002)\end{array}$} \\
\hline & $\%$ & IC95\% & $\%$ & IC95\% \\
\hline \multicolumn{5}{|l|}{ Consumo regular } \\
\hline Frutas & 51,5 & $49,1-53,9$ & 44,3 & $43,1-47,3$ \\
\hline Hortaliças & 81,6 & $79,7-83,4$ & 79,6 & $77,1-81,9$ \\
\hline Frutas e hortaliças & 47,1 & $44,7-49,5$ & 39,8 & $37,0-42,7$ \\
\hline \multicolumn{5}{|l|}{ Atividade física no lazer } \\
\hline Ativo & 38,1 & $35,8-40,5$ & 33,5 & $30,8-36,3$ \\
\hline Inativo & 61,9 & $59,5-64,2$ & 66,5 & $63,7-69,2$ \\
\hline \multicolumn{5}{|l|}{ Atividade física ocupacional } \\
\hline Ativo & 13,4 & $11,9-15,2$ & 16,2 & $13,8-18$ \\
\hline Inativo & 86,6 & $84,8-88,1$ & 83,8 & $81-86,2$ \\
\hline \multicolumn{5}{|l|}{ Atividade física no transporte } \\
\hline Ativo & 7,4 & $6,2-8,8$ & 8,5 & $7,0-10,4$ \\
\hline Inativo & 92,6 & $91,5-93,8$ & 91,5 & $90,2-92,7$ \\
\hline 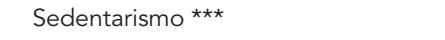 & 29,7 & $27,5-31,9$ & 31,2 & $28,5-34,0$ \\
\hline \multicolumn{5}{|l|}{ Consumo de tabaco } \\
\hline Fumantes & 11,1 & $9,7-12,6$ & 14,7 & $16,2-17,2$ \\
\hline Ex-fumantes & 22,5 & $20,5-24,6$ & 22,1 & $19,8-24,5$ \\
\hline Consumo grave de álcool \# & 15,9 & $14,3-17,8$ & 19,5 & $17,2-22,1$ \\
\hline Consumo de risco de álcool \#\# & 23,2 & $21,2-25,3$ & 25,7 & $23,2-28,4$ \\
\hline \multicolumn{5}{|l|}{ Auto-referência a } \\
\hline Excesso de peso $\left(\mathrm{IMC} \geq 25 \mathrm{Kg} / \mathrm{m}^{2}\right)$ & 18,4 & $34,1-38,9$ & 37,1 & $34,3-40,0$ \\
\hline Obesidade (IMC $\left.\geq 30 \mathrm{Kg} / \mathrm{m}^{2}\right)$ & 36,5 & $9,1-12,2$ & 9,9 & $8,3-11,7$ \\
\hline Hipertensão arterial & 10,6 & $20,4-24,4$ & 19,3 & $17,3-21,4$ \\
\hline Diabetes & 22,4 & $3,5-5,5$ & 3,9 & $2,9-5,1$ \\
\hline Dislipidemias & 4,4 & $16,6-20,4$ & 14,4 & $12,7-16,4$ \\
\hline \multicolumn{5}{|c|}{ * Ajustado para "representar" a população adulta do município com telefone; } \\
\hline \multicolumn{5}{|c|}{ ** Ajustada para "representar" a população adulta total do município; } \\
\hline \multicolumn{5}{|c|}{ 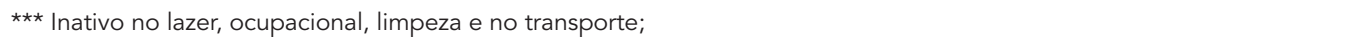 } \\
\hline \multicolumn{5}{|l|}{ \# Consumo grave de álcool $\geq 5$ doses; } \\
\hline nsumo de risco de álcool: mulhe & & & & \\
\hline
\end{tabular}

de frutas e hortaliças e de bebidas alcoólicas diminuíram com o aumento da idade. Foi observada associação inversa entre escolaridade e freqüência do consumo inadequado de frutas e hortaliças, sedentarismo, hipertensão arterial e tabagismo, ou seja, uma maior freqüência desses fatores de risco foi observada para aqueles que apresentavam menor escolaridade. Ao contrário, o excesso de peso e o consumo de bebidas alcoólicas aumentaram com os anos de estudo (Tabela 4).

Para as mulheres, o aumento da idade apresentou associação direta com excesso de peso, hipertensão arterial e tabagismo, já o consumo inadequado de frutas e hortaliças e de bebidas alcoólicas foi maior entre as mais jovens. As mulheres de maior escolaridade apresentaram, acentuadamente, menores freqüências para o excesso de peso, hipertensão arterial e tabagismo (Tabela 5).

\section{Discussão}

A partir de um sistema de monitoramento de fácil operacionalização denominado SIMTEL, observou-se em Goiânia uma alta freqüência de fatores de risco para doenças crônicas não transmissíveis e de doenças crônicas não transmissíveis auto-referidas, que se distribuíram de 
Freqüência de fatores de risco e proteção para doenças crônicas não transmissíveis na população adulta segundo sexo. Goiânia, Goiás, Brasil, 2005.

\begin{tabular}{|c|c|c|c|c|}
\hline \multirow[t]{2}{*}{ Fatores } & \multicolumn{2}{|c|}{ Homens * $(n=763)$} & \multicolumn{2}{|c|}{ Mulheres * $(n=1.239)$} \\
\hline & $\%$ & IC95\% & $\%$ & IC95\% \\
\hline \multicolumn{5}{|l|}{ Consumo regular } \\
\hline Frutas & 43,5 & $39,7-47,5$ & 56,3 & $53,2-59,3$ \\
\hline Hortaliças & 76,4 & $72,9-79,6$ & 84,8 & $82,4-86,9$ \\
\hline Frutas e hortaliças & 38,5 & $34,7-42,4$ & 52,2 & $49,2-55,3$ \\
\hline \multicolumn{5}{|l|}{ Atividade física no lazer } \\
\hline Ativo & 46,8 & $42,9-50,8$ & 32,9 & $30,0-35,8$ \\
\hline Inativo & 53,2 & $49,2-57,1$ & 67,1 & $64,2-70,0$ \\
\hline \multicolumn{5}{|l|}{ Atividade física ocupacional } \\
\hline Ativo & 17,2 & $14,5-20,4$ & 11,2 & $9,3-13,3$ \\
\hline Inativo & 82,8 & $80,0-85,5$ & 88,8 & $86,7-90,7$ \\
\hline \multicolumn{5}{|l|}{ Atividade física no transporte } \\
\hline Ativo & 6,7 & $5,0-8,8$ & 7,8 & $6,3-9,7$ \\
\hline Inativo & 93,3 & $91,2-95,0$ & 92,2 & $90,3-93,7$ \\
\hline Sedentarismo ** & 34,8 & $31,18-38,6$ & 26,6 & $23,9-29,4$ \\
\hline \multicolumn{5}{|l|}{ Consumo de tabaco } \\
\hline Fumantes & 15,1 & $12,5-18,0$ & 8,7 & $7,1-10,5$ \\
\hline Ex-fumantes & 31,0 & $27,4-34,8$ & 17,4 & $15,2-19,9$ \\
\hline Consumo grave de álcool *** & 29,8 & $26,3-33,5$ & 7,6 & $6,2-9,4$ \\
\hline Consumo de risco de álcool \# & 37,7 & $33,9-41,6$ & 14,4 & $12,4-16,7$ \\
\hline \multicolumn{5}{|l|}{ Auto-referência a } \\
\hline Excesso de peso (IMC $\left.\geq 25 \mathrm{Kg} / \mathrm{m}^{2}\right)$ & 45,3 & $41,4-42,9$ & 30,9 & $28,0-33,9$ \\
\hline Obesidade (IMC $\left.\geq 30 \mathrm{Kg} / \mathrm{m}^{2}\right)$ & 11,0 & $8,7-13,7$ & 10,3 & $8,5-12,5$ \\
\hline Hipertensão arterial & 20,0 & $17,1-23,2$ & 23,8 & $21,3-26,5$ \\
\hline Diabetes & 4,3 & $3,0-6,3$ & 4,4 & $3,3-5,9$ \\
\hline Dislipidemias & 14,1 & $11,6-17,1$ & 21,0 & $18,6-23,6$ \\
\hline
\end{tabular}

* Ajustado para "representar" a população adulta do município com telefone;

** Inativo no lazer, ocupacional, limpeza e no transporte;

*** Consumo de álcool $\geq 5$ doses/dia pelo menos 1 vez no último mês;

\# Consumo de risco de álcool: mulher > 1 dose; homem $>2$ doses.

forma diferenciada por sexo, faixa etária e nível de escolaridade.

Neste estudo foram pesquisados, de acordo com a OMS 2, os fatores de risco mais importantes para a morbimortalidade relacionada às doenças crônicas não transmissíveis, vale dizer, a hipertensão arterial sistêmica, hipercolesterolemia, ingestão insuficiente de frutas e hortaliças, excesso de peso, obesidade, inatividade física, tabagismo e consumo de álcool. Além desses fatores de risco, foram incluídos no questionário do SIMTEL aspectos demográficos e sócio-econômicos, que permitem entender a distribuição dos fatores de risco na população e orientar as políticas de saúde a serem desenvolvidas pelo município.

O SIMTEL foi criado tendo como referência o Behavioral Risk Factor Surveillance System
(BRFSS), sistema desenhado para coletar dados usando técnica de telefonia com amostra representativa dos 50 estados e três territórios dos Estados Unidos. A principal proposta deste sistema é fornecer estimativa dos comportamentos de saúde relacionados às principais causas de morbimortalidade entre adultos americanos 16 .

Semelhante ao observado na implantação do SIMTEL no Município de São Paulo (SIMTEL/ São Paulo), no ano de 2003, o SIMTEL/Goiânia, apresenta bons resultados seja quanto à taxa de sucesso na realização das entrevistas $(73,1 \%)$, na agilidade, baixo custo operacional (R\$ 6,20 por entrevista, desconsiderando infra-estrutura englobada permanentemente ao local de pesquisa) e quanto à cobertura da população. Como em São Paulo, os principais motivos para a não-efetivação das entrevistas foram: recusa $(6,8 \% \mathrm{em}$ 
Freqüência de fatores de risco e proteção para doenças crônicas não transmissíveis na população masculina adulta segundo idade e escolaridade. Goiânia Goiás, Brasil, 2005

\begin{tabular}{|c|c|c|c|c|c|c|c|c|c|c|c|c|}
\hline \multirow[t]{2}{*}{ Variáveis } & \multicolumn{2}{|c|}{$\begin{array}{c}\text { Consumo } \\
\text { inadequado } \\
\text { de frutas e } \\
\text { hortaliças * }\end{array}$} & \multicolumn{2}{|c|}{ Sedentarismo ** } & \multicolumn{2}{|c|}{$\begin{array}{c}\text { Excesso } \\
\text { de } \\
\text { peso } \star \star \star\end{array}$} & \multicolumn{2}{|c|}{$\begin{array}{l}\text { Hipertensão } \\
\text { arterial }\end{array}$} & \multicolumn{2}{|c|}{ Tabagismo } & \multicolumn{2}{|c|}{$\begin{array}{c}\text { Consumo } \\
\text { de } \\
\text { álcool \# }\end{array}$} \\
\hline & $\%$ & $p^{\# \#}$ & $\%$ & $p^{\# \#}$ & $\%$ & $p^{\# \#}$ & $\%$ & $p^{\# \#}$ & $\%$ & $p^{\# \#}$ & $\%$ & p\#\# \\
\hline \multicolumn{13}{|l|}{ Idade (anos) } \\
\hline $18-24$ & 68,4 & $<0,01$ & 27,6 & $<0,01$ & 25,3 & $<0,01$ & 4,2 & $<0,01$ & 12,7 & 0,35 & 46,0 & $<0,01$ \\
\hline $25-34$ & 69,6 & & 32,6 & & 43,8 & & 5,6 & & 15,6 & & 49,6 & \\
\hline $35-44$ & 57,2 & & 42,6 & & 54,3 & & 11,4 & & 11,3 & & 31,2 & \\
\hline $45-54$ & 59,9 & & 28,3 & & 61,9 & & 34,8 & & 18,5 & & 39,9 & \\
\hline $55-64$ & 57,1 & & 34,2 & & 42,6 & & 42,5 & & 22,7 & & 26,0 & \\
\hline $65 e+$ & 45,8 & & 52,2 & & 43,0 & & 55,8 & & 11,7 & & 17,4 & \\
\hline Escolaridade (anos) & & $<0,01$ & & 0,02 & & 0,01 & & $<0,01$ & & $<0,001$ & & $<0,01$ \\
\hline $0-4$ & 71,2 & & 43,7 & & 39,8 & & 35,7 & & 23,2 & & 19,9 & \\
\hline $5-8$ & 70,2 & & 40,7 & & 46,7 & & 15,2 & & 23,7 & & 40,9 & \\
\hline $9-11$ & 60,3 & & 33,7 & & 42,4 & & 17,3 & & 11,0 & & 43,3 & \\
\hline 12 e + & 48,8 & & 24,9 & & 52,3 & & 17,5 & & 8,0 & & 38,3 & \\
\hline
\end{tabular}

Ajustado para "representar" a população adulta do município com telefone.

* Consumo inadequado: consumo menor que cinco dias da semana de frutas e hortaliças;

** Inativo no lazer, ocupacional, limpeza e no transporte;

*** Inclui pré-obesidade e obesidade;

\# Consumo de risco de álcool: mulher > 1 dose; homem > 2 doses;

\#\# p de tendência linear.

Goiânia contra 6,3\% em São Paulo) e linhas sem sucesso de contato após dez chamadas realizadas em dias e períodos variados (Goiânia 4,4\% e São Paulo 2,9\%) 13 .

Segundo Monteiro et al. 13, na avaliação da representatividade da amostra de indivíduos estudada pelo sistema de monitoramento por meio de entrevistas telefônicas devem-se levar em conta dois aspectos: o primeiro é quanto essa amostra representa o conjunto de indivíduos com telefone (devendo considerar a qualidade do cadastro de linhas telefônicas utilizado para o sorteio das linhas residenciais, a adequação do sistema de amostragem empregado para sortear as linhas telefônicas e usuários a serem entrevistados e a proporção de entrevistas completadas diante do total de entrevistas previstas); e o segundo é o grau de cobertura do serviço de telefonia na população que se quer monitorar.

O cadastro de linhas telefônicas de Goiânia contemplava basicamente linhas residenciais e estava estratificado por bairros. A proporção de entrevistascompletadasemrelaçãoaototaldeentrevistas previstas (taxa de sucesso) foi de $73,1 \%$, sendo este valor inferior ao observado para o
SIMTEL/São Paulo no ano de 2003, que foi de $84,7 \%$. Porém a taxa de sucesso observada no presente estudo foi semelhante à observada em inquérito domiciliar realizado em Goiânia no ano de 2001 (72,9\%) e superior à taxa de sucesso observada pelo sistema BRFSS, que foi de $59 \%$ no ano de 1999 16. Considerando os aspectos acima mencionados, observa-se que a amostra apresenta uma boa representatividade do conjunto de indivíduos com telefone.

Com relação ao grau de cobertura do serviço de telefonia na população, temos que o cadastro de linhas telefônicas residenciais utilizadas pelo SIMTEL/Goiânia continha 307.301 linhas, reduzindo-se deste total o percentual de linhas empresariais $(7,9 \%)$ e considerando o número médio apurado de linhas por domicílio $(1,0)$, chega-se a 279.365 domicílios servidos por pelo menos uma linha telefônica, o que significaria uma cobertura de $72,7 \%$ dos domicílios permanentes estimados para o mesmo ano de 2005, segundo projeções do IBGE.

A comparação entre as características sócio-demográficas dos adultos estudados pelo SIMTEL/Goiânia, com telefone e as características da população adulta total de Goiânia, ana- 
Freqüência de fatores de risco e proteção para doenças crônicas não transmissíveis na população feminina adulta segundo idade e escolaridade. Goiânia, Goiás, Brasil, 2005

\begin{tabular}{|c|c|c|c|c|c|c|c|c|c|c|c|c|}
\hline \multirow[t]{2}{*}{ Variáveis } & \multicolumn{2}{|c|}{$\begin{array}{c}\text { Consumo } \\
\text { inadequado } \\
\text { de frutas e } \\
\text { hortaliças * }\end{array}$} & \multicolumn{2}{|c|}{ Sedentarismo ** } & \multicolumn{2}{|c|}{$\begin{array}{c}\text { Excesso } \\
\text { de } \\
\text { peso *** }\end{array}$} & \multicolumn{2}{|c|}{$\begin{array}{c}\text { Hipertensão } \\
\text { arterial }\end{array}$} & \multicolumn{2}{|c|}{ Tabagismo } & \multicolumn{2}{|c|}{$\begin{array}{c}\text { Consumo } \\
\text { de } \\
\text { álcool \# }\end{array}$} \\
\hline & $\%$ & $p$ & $\%$ & $p$ & $\%$ & $p$ & $\%$ & $p$ & $\%$ & $\mathrm{p}$ & $\%$ & $p$ \\
\hline \multicolumn{13}{|c|}{ Idade (anos) } \\
\hline $18-24$ & 60,2 & $<0,01 \# \#$ & 36,4 & 0,24 & 8,1 & $<0,01 \# \#$ & 2,9 & $<0,01 \# \#$ & 3,2 & $<0,01 *$ & 19,4 & $<0,01 \# \#$ \\
\hline $25-34$ & 44,9 & & 22,2 & & 22,5 & & 8,9 & & 6,1 & & 16,6 & \\
\hline $35-44$ & 50,4 & & 24,8 & & 30,6 & & 16,0 & & 9,6 & & 20,4 & \\
\hline $45-54$ & 36,7 & & 23,4 & & 41,3 & & 30,6 & & 15,0 & & 10,1 & \\
\hline $55-64$ & 38,8 & & 23,2 & & 51,2 & & 49,4 & & 9,9 & & 8,0 & \\
\hline $65 e+$ & 22,3 & & 36,5 & & 43,1 & & 64,1 & & 6,2 & & 4,3 & \\
\hline \multicolumn{13}{|c|}{ Escolaridade (anos) } \\
\hline $0-4$ & 43,5 & 0,09 & 23,5 & 0,15 & 47,8 & $<0,01$ \#\# & 46,9 & $<0,01$ \#\# & 13,5 & $<0,01 \# \#$ & 5,6 & $<0,01$ \#\# \\
\hline $5-8$ & 45,7 & & 24,8 & & 40,3 & & 29,0 & & 12,2 & & 13,9 & \\
\hline $9-11$ & 46,9 & & 22,6 & & 24,7 & & 15,9 & & 7,4 & & 18,2 & \\
\hline 12 e + & 35,3 & & 36,4 & & 24,5 & & 15,5 & & 4,6 & & 15,4 & \\
\hline
\end{tabular}

Ajustado para "representar" a população adulta do município com telefone.

* Consumo inadequado: consumo menor que cinco dias da semana de frutas e hortaliças;

** Inativo no lazer, ocupacional, limpeza e no transporte;

*** Inclui pré-obesidade e obesidade;

\# Consumo de risco de álcool: mulher $>1$ dose; homem > 2 doses;

\#\# p de tendência linear.

lisada pelo censo demográfico em 2000, mostra que a amostra estudada possui maior proporção de mulheres, menor proporção de jovens entre 18 e 24 anos de idade e maior proporção de escolaridade igual ou superior a nove anos. Maior representatividade de mulheres e de indivíduos com alta escolaridade e menor representatividade de indivíduos mais jovens também foram observados no SIMTEL/São Paulo e na amostra do BRFSS 13,16.

Sistemas de monitoramento que se baseiam em dados referidos apresentam certas limitações que devem ser consideradas na interpretação dos dados. A acurácia das informações auto-referidas depende do conhecimento dos respondentes de informações relevantes, da capacidade de recordá-las e da fidedignidade ao recordá-las. As evidências mostram que muitas vezes os entrevistados têm a tendência de omitir alguns comportamentos, que podem ser considerados socialmente inaceitáveis tais como tabagismo e excesso de peso. Ao contrário, os entrevistados podem superestimar comportamentos que são desejáveis tais como atividade física e consumo de frutas e hortaliças. Portanto, a situação real da freqüência dos fatores de risco para doenças crônicas não transmissíveis possivelmente é pior do que a relatada.

Apesar das limitações apontadas, o sistema de monitoramento por ligações telefônicas possibilita monitorar a carga e a tendência das doenças crônicas não transmissíveis no nível local, apoiando o planejamento, implementação e avaliação de intervenções em saúde pública, voltadas para a redução destas doenças. Permite também testar hipóteses já estabelecidas e propor outras para serem analisadas posteriormente, em estudos com delineamentos mais apropriados. Além desses aspectos, destaca-se pela facilidade na obtenção das informações a partir de uma amostra representativa sem grande custo, permitindo sua realização de forma sistemática.

No presente estudo, observou-se uma freqüência relativamente alta, em ambos os sexos, de baixo consumo de frutas e hortaliças, sedentarismo, excesso de peso e hipertensão. Para o consumo de frutas e hortaliças, foi observado que $52,9 \%$ da população estudada apresentaram uma freqüência de consumo menor que cinco vezes na semana sendo esta bem inferior ao recomendado pela estratégia global para uma alimentação saudável 17. Chama atenção 
que mesmo entre os indivíduos de maior escolaridade o consumo regular de frutas e hortaliças foi baixo.

Quando considerado o conjunto das atividades físicas que geram gasto energético, atividade física ocupacional, de lazer, no trabalho doméstico e como meio de locomoção, aproximadamente $50 \%$ da população foi classificada como sedentária. Quanto ao sedentarismo nos momentos de lazer, a freqüência foi semelhante ao observado em estudo anterior realizado em Goiânia (61,9\%) 17. Assim como em outros estudos, as mulheres se apresentaram mais sedentárias que os homens, e a prática de atividade física diminuiu com a idade 18,19.

Os homens apresentaram maiores prevalências de tabagismo $(15,1 \%)$ e de consumo de álcool $(29,8 \%)$ do que as mulheres $(8,7 \%$ e $7,6 \%$, respectivamente); em ambos os sexos, a prevalência de tabagismo foi maior nos indivíduos com menor escolaridade (ensino fundamental completo ou incompleto), quando comparado com os de maior escolaridade. Esses achados são consistentes com os relatados pela literatura para o Brasil e para países em desenvolvimento 11,14.

Neste estudo, a prevalência de excesso de peso observada para os homens $(45,3 \%)$ e para as mulheres $(30,9 \%)$ foi superior e inferior, respectivamente, ao observado em estudos recentes em âmbito nacional 8,14 e em estudo epidemiológico realizado em 2001 no Município de Goiânia, no qual a prevalência de excesso de peso foi de $41,9 \%$ para os homens e de $43 \%$ para as mulheres 20 . Tais diferenças entre as estimativas do SIMTEL e o dado de prevalência para excesso de peso disponível para Goiânia ocorreram em parte pelas características próprias deste tipo de sistema, em que as medidas são passíveis de vieses decorrentes do fato de serem auto-referidas, além de obtidas por telefone.

O controle da qualidade dos dados obtidos no SIMTEL foi realizado pela avaliação da gravação das entrevistas e rechamada telefônica quando necessário. Estudos de validade e confiabilidade do sistema foram realizados no mesmo período em São Paulo, mostrando a viabilidade do sistema, segundo relato dos autores. Tais estudos estão em vias de publicação.

As características da amostra do SIMTEL (homens e mulheres de escolaridade superior à da população geral), que em nosso meio reconhecidamente favorecerem a uma maior prevalência de excesso de peso entre os homens e menor prevalência entre as mulheres, também contribuíram para os vieses observados, fato que se procurou controlar pelo uso do fator de expansão para a população total. O intervalo de tempo decorrido (3,5 anos) entre os dois estudos explica melhor as diferenças, que seguem a tendência nacional 22 .

A prevalência de hipertensão foi semelhante à observada em outros estudos que também utilizaram a auto-referência do diagnóstico médico de hipertensão, porém esta prevalência foi subestimada em relação à prevalência obtida por meio de medições no ano de 2001 no Município de Goiânia. Isto ocorre em grande parte pelo fato de que cerca de $50 \%$ dos hipertensos desconhecem sua condição 22 .

A prevalência de diabetes foi inferior, contudo próxima aos valores encontrados na campanha nacional de detecção de suspeitos de diabetes mellitus, realizada no Brasil em 2001, em adultos com 40 anos e mais, em que a prevalência variou de 5,2\% em Brasília a 9,7\% em São Paulo.

Alguns estudos mostram melhor acurácia para auto-referência de diabetes do que para outras doenças crônicas. O contraste com a hipertensão confirma um importante resultado observado em estudos prévios nos quais a acurácia de autoreferência variou grandemente entre as condições crônicas, e esta precisão correlacionou-se com a idade, escolaridade, tempo de exame de saúde mais recente e função cognitiva 23 .

A comparação entre estimativas nacionais da prevalência de sobrepeso e obesidade pelo BRFSS e pelo National Health and Nutrition Examination Survey (NHANES) mostra que no BRFSS a prevalência da obesidade foi subestimada em $9,5 \%$ e o sobrepeso em $5,7 \%$; todavia essa subestimação não ocorreu de forma sistemática entre os diferentes grupos demográficos. Os autores deste estudo concluíram que, apesar de não classificar corretamente a prevalência de sobrepeso e obesidade entre os diferentes grupos demográficos, o BRFSS foi capaz de hierarquizar corretamente as populações de acordo com a carga de obesidade e sobrepeso 16

Perante os resultados observados, fica evidente que para reduzir os fatores de risco para a morbimortalidade decorrente das doenças crônicas não transmissíveis na população adulta de Goiânia são necessárias estratégias de prevenção e promoção da saúde, tais como estímulo à prática regular de atividade física e ao consumo adequado de frutas e hortaliças, redução na freqüência do excesso de peso, consumo excessivo de bebidas alcoólicas e do tabagismo, medidas que reconhecidamente previnem as doenças crônicas não transmissíveis. O SIMTEL/Goiânia poderá monitorar a efetividade das estratégias e colaborar com a estratégia global para alimentação saudável, atividade física e saúde da Organização Mundial da Saúde (EG/OMS) no referente à recomendação de monitoramento e vigilância das doenças crônicas não transmissíveis. 


\section{Resumo}

Este estudo descreve alguns resultados do sistema de monitoramento de fatores de risco para doenças crônicas por entrevistas telefônicas no Município de Goiânia, Goiás, Brasil, 2005. Foi estudada amostra probabilística $(n=2.002)$ da população adulta servida por linhas telefônicas residenciais fixas. Foram analisadas variáveis comportamentais (consumo alimentar, atividade física, tabagismo e consumo de bebida alcoólica), peso e altura referidos e referência a diagnóstico médico de doenças crônicas. Foram calculadas estimativas de prevalência e valores de qui-quadrado. Observou-se baixo consumo de frutas e hortaliças $(47,1 \%)$, alta freqüencia de inatividade física ocupacional (86,6\%), no deslocamento para o trabalho (92,6\%) e lazer (61,9\%), consumo excessivo de bebidas alcoólicas (23,2\%), excesso de peso (36,5\%), obesidade (10,6\%), hipertensão arterial (22,4\%), dislipidemias (18,4\%) e diabetes $(4,4 \%)$. A maioria dos fatores de risco apresentou associação inversa com escolaridade e direta com idade, com diferenças significativas entre sexos $(p<0,05)$. Observou-se alta prevalência dos fatores de risco de doenças crônicas não transmissíveis e de auto-referidas. Aspectos positivos do sistema: baixo custo operacional, possibilidade de monitorar a carga e a tendência das doenças crônicas não transmissíveis no nível local.

Doença Crônica; Monitoramento; Fatores de Risco; Entrevistas; Telefone

\section{Colaboradores}

M. R. G. Peixoto, V. P. Alexandre e E. C. Moura organizaram o banco de dados e executaram as análises estatísticas. V. P. Alexandre e R. G. M. Souza contribuíram com a coleta de dados. M. R. G. Peixoto, R. G. M. Souza, V. P. Alexandre e E. T. Monego foram responsáveis pela fundamentação teórica e elaboração do texto.

\section{Agradecimentos}

Órgão financiador: Conselho Nacional de Desenvolvimento Científico e Tecnológico (CNPq Edital CT - Saúde/MCT/MS/CNPq nº. 30/2004 - Alimentação e Nutrição).

\section{Referências}

1. Coordenação Geral da Política de Alimentação e Nutrição, Secretaria de Assistência à Saúde, Ministério da Saúde. Guia alimentar para a população brasileira: promovendo a alimentação saudável. Brasília: Ministério da Saúde; 2005. (Série A. Normas Técnicas e Manuais).

2. World Health Organization. Diet, nutrition and prevention of chronic diseases. Geneva: World Health Organization; 2003.

3. World Health Organization. The world health report 2002: reducing risks, promoting healthy life. Geneva: World Health Organization; 2002.

4. Monteiro CA, Mondini L, Levi-Costa RB. Mudanças na composição e adequação nutricional da dieta familiar nas áreas metropolitanas do Brasil (1988 -1996). Rev Saúde Pública 2000; 34:251-8.

5. Mahoney LT, Clarke WR, Burns TL, Lauer RM. Childhood predictors of high blood pressure. Am J Hypertens 1991; 4:608-10.
6. Li S, Chen W, Srinivasan SR, Bond M. Childhood cardiovascular risk factors and carotid vascular changes in adulthood: The Bogalusa Heart Study. JAMA 2003; 290:2271-6.

7. Lopes ACS, Calaffa WT, Sichieri R, Mingoti SA, Lima-Costa MF. Consumo de nutrientes em adultos e idosos em estudo de base populacional: Projeto Bambuí. Cad Saúde Pública 2005; 21:1201-9.

8. Monteiro CA, Conde WL, Popkin BM. Is obesity replacing or adding to under-nutrition? Evidence from different social classes in Brazil. Public Health Nutr 2002; 5:251-8.

9. Marcopito LF, Rodrigues SSF, Pacheco MA Shirassu MM, Goldfeder AJ, Moraes MA. Prevalência de alguns fatores de risco para doenças crônicas na cidade de São Paulo. Rev Saúde Pública 2005; 39:738-45. 
10. Marques APO, Arruda IKG, Santo ACGE, Raposo MCF, Guerra MD, Sales TF. Prevalência de obesidade e fatores associados em mulheres idosas. Arq Bras Endocrinol Metabol 2005; 49:441-8.

11. World Health Organization. Guidelines for controlling and monitoring the tobacco epidemic. Geneva: World Health Organization; 1998.

12. Silveira EA, Araújo CL, Gigante DP, Barros AJD, Lima MS. Validação do peso e altura referidos para o diagnóstico do estado nutricional em uma população de adultos no Sul do Brasil. Cad Saúde Pública 2005; 21:235-45.

13. Monteiro CA, Moura EC, Jaime PC, Lucca A, Florindo AA, Figueiredo ICR, et al. Monitoramento de fatores de risco para doenças crônicas por meio de entrevistas telefônicas: métodos e resultados no município de São Paulo. Rev Saúde Pública 2005; 39:47-57.

14. Instituto Nacional de Câncer. Inquérito domiciliar sobre comportamentos de risco e morbidade referida de doenças e agravos não transmissíveis: Brasil, 15 capitais e Distrito Federal, 2002-2003. Rio de Janeiro: Instituto Nacional de Câncer; 2004.

15. World Health Organization. Physical status: the use and interpretation of anthropometry. Geneva: World Health Organization; 1995.

16. Centers for Disease Control and Prevention. Behavioral Risk Factor Surveillance System - BRFSS. http://www.cdc.gov/brfss (acessado em 02/ Out/2006)
17. Organización Mundial de la Salud. Estrategia mundial sobre régimen alimentario, actividad física y salud. In: 57a Asamblea Mundial de la Salud. http://www.who.int/es/ (acessado em 10/ Out/2006).

18. World Health Organization. World health day 2002. Sedentary lifestyle: a global public health problem. http://www.who.int/world-health-day/ (acessado em 10/Out/2006).

19. Hallal PC, Victora CG, Wells JC, Lima RC. Physical inactivity: prevalence and associated variables in Brazilian adults. Med Sci Sports Exerc 2003; 35:1894-900

20. Peixoto MRG. Estudo de índices antropométricos na população adulta de Goiânia [Tese de Doutorado]. São Paulo: Faculdade de Saúde Pública, Universidade de São Paulo; 2004.

21. Ministério da Saúde. Vigilância de fatores de risco e proteção para doenças crônicas por inquérito telefônico. Brasília: Ministério da Saúde; 2007.

22. Peixoto MRG, Benício MHA, Latorre MRD, Jadim PCBV. Circunferência da cintura e índice de massa corporal como preditores da hipertensão arterial. Arq Bras Cardiol 2006; 87:462-70.

23. Lima-Costa MF, Peixoto SV, Firmo JOA. Validade da hipertensão arterial auto-referida e seus determinantes (Projeto Bambuí). Rev Saúde Pública 2004 38:637-42.

Recebido em 08/Jan/2007

Versão final reapresentada em 05/Out/2007

Aprovado em 25/Out/2007 